\title{
Segregation in granular matter under horizontal swirling excitation
}

\author{
S. Aumaître, C. A. Kruelle, and I. Rehberg \\ Experimentalphysik V, Universität Bayreuth, D-95440 Bayreuth, Germany
}

(Received 19 December 2000; published 26 September 2001)

\begin{abstract}
A segregation phenomenon in a horizontally vibrated monolayer of granular matter is studied experimentally. In a binary mixture of small spheres and larger disks, the collapse speed of the disks increases dramatically with increasing granular temperature. The scaling behavior can be understood by applying arguments from kinetic gas theory.
\end{abstract}

DOI: 10.1103/PhysRevE.64.041305

PACS number(s): 45.70.Mg, 05.20.-y, 64.75.+g

Solid particles of different sizes tend to demix when moved. This effect occurs in Nature in rock avalanches [1], for example, and is of tremendous importance in industrial powder processing [2]. Recently, a particular impressive phenomenon of size segregation became known under the name Brazil nut effect [3,4].

Experimental investigations of related phenomena include an inclined plane where the binary mixture flows under gravity. Larger particles accumulate at the front of the avalanche, which then shows a remarkable fingering instability [5]. A half-filled rotating drum is often used to study this phenomenon in a continuous flow. Both radial [6] and axial [7] segregations - with very different time scales-occur in this system. Segregation under vertical shaking is also extensively studied, because an interesting counterintuitive effect occurs where the large particles end up in a layer on top of the small ones $[8,9]$. Qualitative explanations for these phenomena involve size effects, convection, or the friction difference between both particle species [6-10].

Here we describe segregation in a particularly simple model system, namely, a monolayer of granular matter driven by isotropic horizontal motion. This system is attractive because theoretical ideas about the equilibrium thermodynamics of hard spheres can be adapted to understand the segregation: Due to the hard sphere interaction, there exists an excluded volume for the small particles around a large particle. This excluded volume becomes smaller when large particles are in contact. This means an entropy gain for the small spheres. The corresponding free energy grows linearly with the ratio of the particle sizes due to depletion [11]. From an experimental point of view an advantage of our two-dimensional system is the fact that particle motion can be traced easily.

We study segregation with a device similar to that described in Ref. [12]. A circular plexiglass ring of $19-\mathrm{cm}$ inner diameter is put on a glass plate mounted on top of a platform shaker. The excitation can be described as a horizontal swirling motion, i.e., a superposition of two sinusoidal vibrations in $x$ and $y$ directions which results in a circular motion of the entire platform with a radius $A$ of $1 \mathrm{~cm}$ and variable rotation frequency $\nu$ in the range of $0.43-2 \mathrm{~Hz}$. A charge-coupled-device (CCD) camera is fixed over the container in the comoving frame. The images are digitized with a resolution of $256 \times 256$ pixels each $0.02 \mathrm{~s}$.

The container is filled with a binary mixture of 600 small transparent glass spheres (diameter $d=4 \mathrm{~mm}$, mass $m$
$=0.08 \mathrm{~g}$ ) and 19 aluminum disks (diameter $D=10 \mathrm{~mm}$, height $h=6 \mathrm{~mm}$, mass $M=1.64 \mathrm{~g}$ ). The static friction coefficient $\mu_{s}$ of the disks has been measured on an inclined plane to be about $0.43 \pm 20 \%$, and the kinetic friction coefficient $\mu_{k}$ to be about $0.27 \pm 20 \%$. The restitution coefficient for sphere-cylinder collision is estimated to be about 0.8 following Foerster et al. [13].

The bottom plate of the container is leveled with a precision of $2 \mathrm{mrad}$, and is coated with an electrically conducting indium tin oxide layer to suppress static electricity. A sheet of paper is glued at the bottom of the cylinders to prevent scratches in this layer.

In order to obtain reproducible measurements, we always start with the same initial condition: The disks are placed on a hexagonal lattice with $3-\mathrm{cm}$ spacing (i.e., larger than the driving amplitude) as indicated by the large black disks in Fig. 1(a). Figure 1(b) is a snapshot of the experiment $360 \mathrm{~s}$ after the start of the shaking. The clustering of the large particles is more evident after 4896 s, as indicated in Fig. 1(c). At this driving frequency a completely clustered state occurs only after more than $20 \mathrm{~h}$.

The positions of the 19 large disks are captured every $0.02 \mathrm{~s}$ by the computer. (For this purpose it turned out to be necessary to use transparent small spheres, not the black spheres used for the snapshot of Fig. 1). From these, at each acquisition time, we extracted (i) the number of pair contacts between collapsed particles, $N_{c}$, which can evolve from 0 to 42 (when all 19 particles are collapsed into one symmetric hexagonal cluster); and (ii) for each particle the distance to its nearest neighbor, $l_{\mathrm{nn}}$. The mean $L_{\mathrm{nn}}=\left\langle l_{\mathrm{nn}}\right\rangle$ over the 19 large particles is a useful and reproducable measure to characterize segregation dynamics before the collapsed phase is reached. The standard deviation $\sigma_{\mathrm{nn}}=\sqrt{\left\langle l_{\mathrm{nn}}^{2}-L_{\mathrm{nn}}^{2}\right\rangle}$ provides
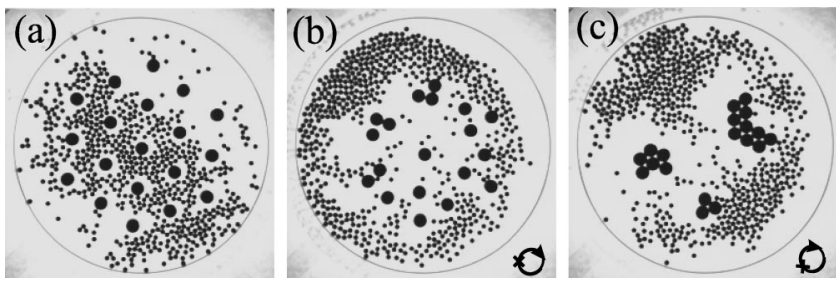

FIG. 1. 19 disks and 600 spheres observed in a comoving frame driven with $1.16 \mathrm{~Hz}$. (a) Initial arrangement. (b) After $360 \mathrm{~s}$. (c) After $4896 \mathrm{~s}$. The black circles indicate the motion of the surface in the laboratory frame. 


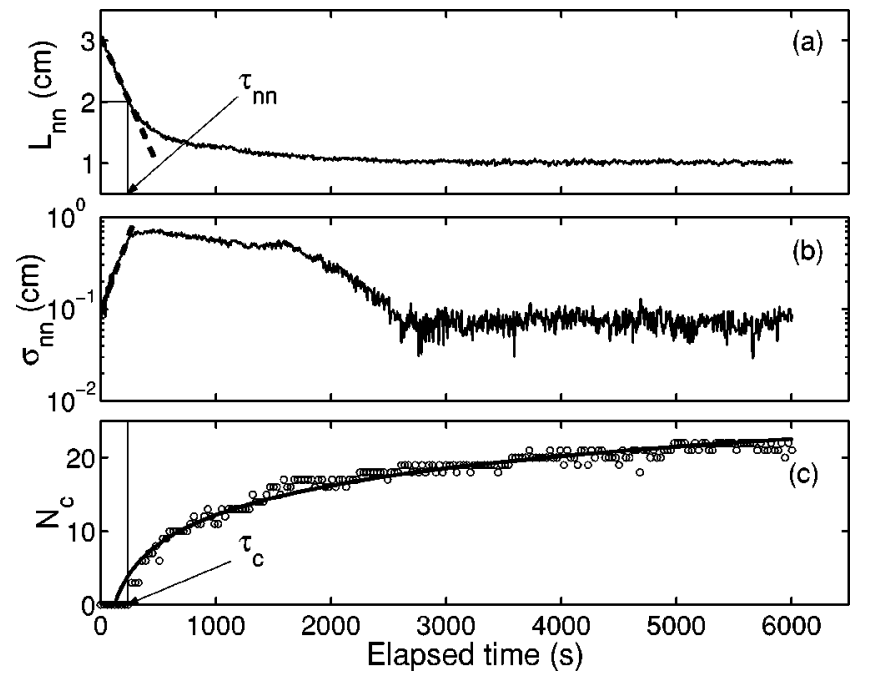

FIG. 2. Evolution of (a) the distance $L_{\mathrm{nn}}$ (solid line) and the fit to the initial linear decay (dashed line); (b) the standard deviation $\sigma_{\mathrm{nn}}$ in a semilogarithmic axis and the fit for the exponential growth (dashed line); and (c) the number of contacts $N_{c}(\bigcirc)$ and the fit for logarithmic growth (solid line). The driving frequency is $\nu=1.16$ $\mathrm{Hz}$.

information about the disorder of the disk positions.

Figure 2(a) shows the temporal evolution of $L_{\mathrm{nn}}(t)$ for a driving frequency $\nu=1.16 \mathrm{~Hz}$. This value decreases from 3 $\mathrm{cm}$ (the initial distance on the hexagonal lattice) to an asymptotic value of about $1 \mathrm{~cm}$ (the minimal distance for particles in contact). This curve can be characterized by the slope of the initial linear decay, indicated by the dashed line. This slope defines a mean collapse velocity, $V_{\mathrm{nn}}=\left[L_{\mathrm{nn}}(0)\right.$ $\left.-L_{1}\right] / \tau_{\mathrm{nn}}$. We measure this within the time interval $\tau_{\mathrm{nn}}$, the time it takes to reach a mean distance of $L_{1}=\left[L_{\mathrm{nn}}(0)\right.$ $\left.+L_{\mathrm{nn}}(\infty)\right] / 2(\approx 2 \mathrm{~cm})$.

Figure 2(b) shows the standard deviation, $\sigma_{\mathrm{nm}}(t)$, of $l_{\mathrm{nn}}$ in a semilogarithmic plot. Initially, this quantity is small since we start with a regular arrangement. First it increases exponentially when disks approach each other. Then it reaches a plateau around a maximum value. It decreases exponentially during the clustering phase, until it reaches the spatial resolution of our device $(\approx 1$ pixel, i. e., $\approx 0.08 \mathrm{~cm})$.

Figure 2(c) shows the temporal evolution of the number of contacts between collapsed particles $N_{c}(t)$. Initially, this is reminiscent of a logarithmic growth, but its value must be bounded by 42 . From this curve we extract the time $\tau_{c}$ at which the first contact occurs, which serves to define the second characteristic collapse velocity $V_{c}$ of disks by $V_{c}$ $=\left[L_{\mathrm{nn}}(0)-D\right] / \tau_{c}$.

The influence of the initial configuration of the disks on the final state has been checked. We observed no qualitative differences between a random arrangement and the hexagonal initial arrangement, provided that the mean distance between nearest neighbors was similar. In addition to $V_{n n}$ and $V_{\mathrm{c}}$, we also tested three other quantities as measures for the speed of the collapse: (i) the number of clusters, (ii) the mean, and (iii) the maximum number of disks per cluster. It turned out that the statistical fluctuations were too strong to capture the dynamics of the clustering process.

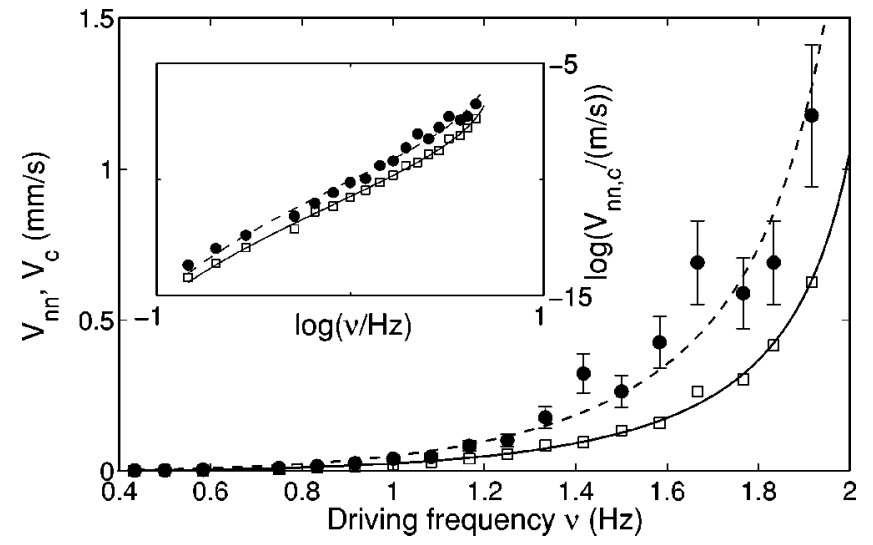

FIG. 3. Evolution of the two characteristic velocities $V_{\mathrm{nn}}(\square)$ and $V_{c}(\bigcirc)$ with the driving frequency $\nu$. The solid and dashed lines represent fits according to Eq. (1). The inset shows the same data on a logarithmic (base 10) scale.

$V_{\mathrm{nn}}$ and $V_{c}$ were measured within a range of driving frequencies $\nu$ from 0.43 to $2 \mathrm{~Hz}$. The results are presented in Fig. 3.

The figure shows-both on linear and logarithmic axesthat $V_{\mathrm{nn}}$ and $V_{c}$ increase much more strongly than linearly with the table velocity $2 \pi A \nu$. No results are shown below a driving frequency of $0.43 \mathrm{~Hz}$. For such small driving accelerations, small spheres do not roll continuously because of the plate roughness. Above $2 \mathrm{~Hz}$, the characteristic times $\tau_{\mathrm{nn}}$ and $\tau_{c}$ are similar to the time that the swirling table requires to reach a stationary motion. This upper limit is still smaller than the sliding threshold $\nu_{s}$ of the disks, which is expected to be $\sqrt{\mu_{s} g / A} / 2 \pi$, and has been measured to be $2.3 \mathrm{~Hz}$ $\pm 20 \%$.

In order to understand the physical mechanism for the collapse, we studied the dynamics of the small spheres in more detail. To this end, 19 transparent plexiglass disks were glued onto the initial lattice, and one of the 600 small spheres was colored black and used as a tracer particle. Since the disks are fixed in their position, ergodicity can be assumed: The temporal trace of one individual black particle represents the density and the velocity distribution of all particles.

Following kinetic gas theory, the spatial distribution of the kinetic energy is related to the pressure distribution: We define the local pressure as $P(\mathbf{r})=n(\mathbf{r}) m v_{\mathrm{rms}}^{2}(\mathbf{r}) / 2$, where $n(\mathbf{r})$ is the surface density of small spheres at a point $\mathbf{r}$, and $v_{\text {rms }}(\mathbf{r})$ the root mean square velocity obtained by temporal averaging over $5 \mathrm{~h}$ at this location. (Note that this definition is a two-dimensional one: A force can be calculated from this pressure by multiplication with a length - not with an area as in the usual definition.)

The resulting pressure distribution of the small spheres is shown as a gray-scale plot in Fig. 4. The observed maximal value of the pressure $P_{\max }=0.016 \mathrm{~J} / \mathrm{m}^{2}$ corresponds to a mean velocity of the small spheres of about 1.2 times the table velocity at this point. Zero pressure is represented in white; the locations of the fixed disks are thus clearly observable. The figure shows that this granular-gas pressure is 


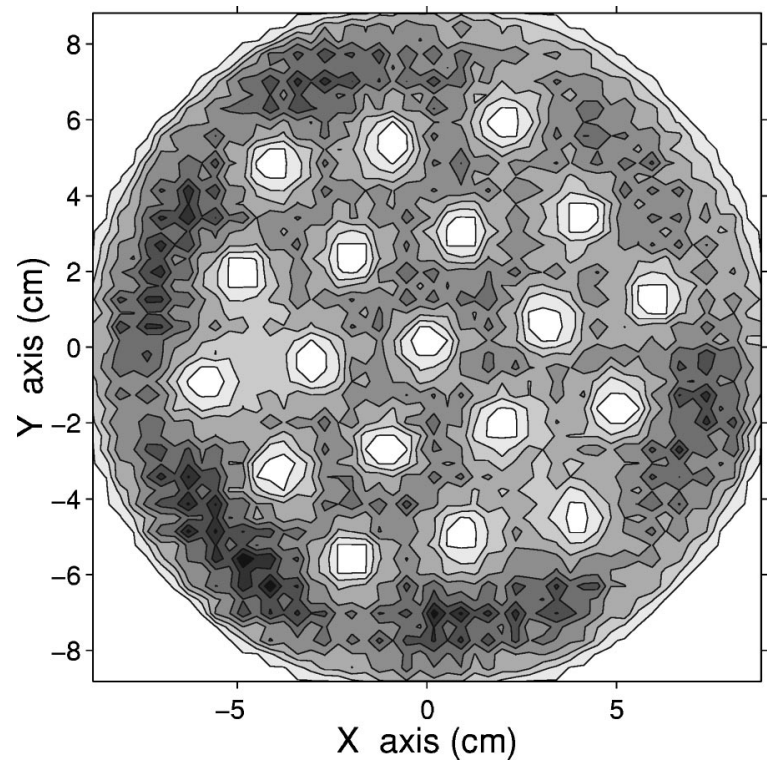

FIG. 4. Pressure density distribution of small spheres with a fixed configuration of the cylindrical disks (white spots) at a swirling frequency $\nu=1.17 \mathrm{~Hz}$. The velocities and densities have been averaged on a grid of $4 \times 4$ pixels over a time period of $5 \mathrm{~h}$. The darkest regions represent a maximum of $0.016 \mathrm{~J} / \mathrm{m}^{2}$.

smaller between the cylinders than outside the array. It is surprising that the spheres do not follow the pressure gradient, i.e., do not increase their density within the array. Obviously, this tendency must be counterbalanced by the interaction with the disks. They must provide a mean force on the spheres, pointing in the radial direction of the container. It is the reaction of this force which tends to drive the clustering when the disks are not fixed.

In the following we present quantitative arguments for the strong increase of the clustering speed of disks with the driving speed of the table, as shown in Fig. 3. The mean disk velocity $V_{d}$ is basically determined by two factors: The frequency of collisions $f$ with small particles, and the distance $\Delta X$ which a disk slides after being struck by a small particle: $V_{d}=\Delta X f$. Both factors depend on the driving frequency. The number of collisions is proportional to the small sphere velocity, which is proportional to the driving angular velocity $\omega=2 \pi \nu$. Since the kinetic friction force $F_{\mathrm{kf}}$ is assumed to be constant during the sliding time, the sliding distance $\Delta X$ is proportional to the energy of the small striking particle, i.e., $\Delta X \propto F_{\mathrm{kf}}^{-1} \omega^{2}$. Because the disks slide on an accelerated table they "feel" an effective force $F_{\mathrm{kf}} \propto \omega_{k}^{2}-\omega^{2}$, where $\omega_{k}$ is the angular velocity of the table for which the inertial force has the same value as the kinetic friction force. At this driving frequency the disks would not stop, and the sliding distance would become infinite. The sliding distance thus increases with the driving frequency: $\Delta X \propto \omega^{2} /\left(\omega_{k}^{2}\right.$ $\left.-\omega^{2}\right)$.

An additional difficulty arises: Not all small particles have sufficient energy to overcome the static friction. The fraction of spheres with sufficient kinetic energy is given by the Boltzmann factor. We thus introduce an effective collision frequency

$$
f_{\mathrm{e}}=f \exp \left(-C_{1} \frac{\left(\omega_{s}^{2}-\omega^{2}\right)^{2}}{\omega^{2}}\right)
$$

The denominator in the exponent represents the granular temperature. The numerator represents the activation energy. This expression can be justified as follows: We assume that the force during the impact of a small sphere colliding with a resting cylinder must overcome the static friction force: $\Delta p / \Delta t>F_{\mathrm{sf}}$. For Hertzian contact the collision time $\Delta t$ is almost constant in the range of velocities considered here [14]. Thus one can postulate a critical threshold velocity $v_{c}$ $\propto F_{\text {sf }}$. The corresponding activation energy is therefore $\propto F_{\text {sf }}^{2}$, which explains the square in the numerator. This energy depends on the driving frequency, since the inertial force $A \omega^{2}$ reduces the threshold force. Here $\omega_{s}$ is the angular velocity of the table at which the inertial force has the same value as the static friction force. At the corresponding driving frequency the disks start to move without impact from the small spheres.

Combining the assumptions about the sliding distance and the effective collision frequency, we finally arrive at

$$
V_{d}=c_{2} \frac{\omega^{3}}{\omega_{k}^{2}-\omega^{2}} \exp \left(-c_{1} \frac{\left(\omega_{s}^{2}-\omega^{2}\right)^{2}}{\omega^{2}}\right),
$$

with $\omega_{k}^{2} / \omega_{s}^{2}=\mu_{k} / \mu_{s}$. The solid and dashed lines in Fig. 3 represent three-parameter fits of this equation to the data. The first parameter $\omega_{k}$ is equal to $13.5 \mathrm{rad} / \mathrm{s}$ for the $V_{c}$ data, and $13.6 \mathrm{rad} / \mathrm{s}$ for $V_{\mathrm{nn}}$. From our measurement of the sliding coefficient on an inclined plane, we expect $\omega_{\mathrm{k}}=15$ $\pm 1 \mathrm{rad} / \mathrm{s}$.

The coefficient $c_{1}=1.1 \times 10^{-4}(\mathrm{~s} / \mathrm{rad})^{2}$ for the $V_{c}$ data, and $c_{1}=1.0 \times 10^{-4}(\mathrm{~s} / \mathrm{rad})^{2}$ for $V_{\mathrm{nn}}$. With the estimate of the activation energy presented above, and the assumption that the granular temperature is $m(\omega A)^{2} / 2$ (which has been checked experimentally, and turned out to be correct within $10 \%$ ), the time $\Delta t$ can be estimated as $1 \mathrm{~ms}$. This seems fairly realistic for the relaxation time of rolling particles [15]. From $c_{1}$ one can also estimate that the multiplicative Boltz-

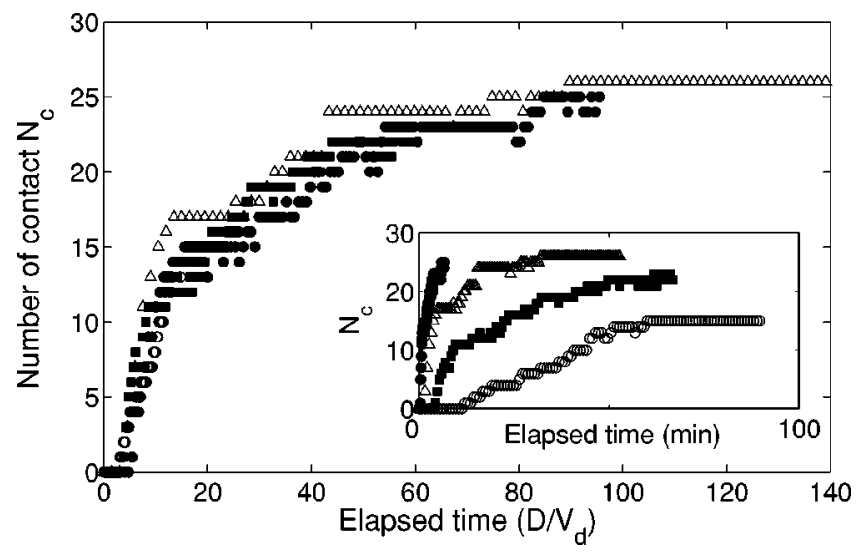

FIG. 5. Number of contacts $N_{c}$ as a function of a rescaled time for the driving frequencies $\nu=1 \mathrm{~Hz}(\bigcirc), \nu=1.33 \mathrm{~Hz}(\boldsymbol{\square})$, $\nu=1.67 \mathrm{~Hz}(\triangle)$, and $\nu=2 \mathrm{~Hz}(\bullet)$. The inset shows $N_{c}$ as a function of time. 
mann factor changes dramatically within the explored frequency range, namely, from $2 \%$ to $98 \%$.

The third fit parameter $c_{2}$ turns out to be $16 \mu \mathrm{m}$ for the $V_{\text {nn }}$ curve, and $32 \mu \mathrm{m}$ for $V_{\mathrm{c}}$. These numbers signify a mean sliding distance within a time $1 / \omega$. The fact that this distance is smaller then the mean free path of the disks indicates that there is indeed only about one contact per sliding time of a disk, which is a necessary condition for our theoretical estimations of the disk velocity.

Finally, we would like to add an experimental observation which goes beyond the analysis presented so far: The velocity $V_{d}$ not only describes the motion before the first collapse, from which $V_{\mathrm{nn}}$ and $V_{c}$ were determined, but also that during the later stages of the clustering process. In Fig. 5 the number $N_{c}$ of contacts between collapsed particles is plotted as a function of a dimensionless time, which is obtained by mul- tiplication with $V_{d} / D$. All data, taken for different driving frequencies between 1 and $2 \mathrm{~Hz}$, collapse on a single curve.

In summary, we presented experimental measurements of the collapse velocity function. By image processing techniques, we observed a pressure gradient which explains the collapse qualitatively. The strong increase of the collapse velocity with the granular temperature can be understood with statistical assumptions for the motion of the collapsing disks. This velocity also serves to scale the temporal evolution of the cluster size after the first contact. The roles of the finite container size and the particle density need further investigation.

We would like to thank A. Breu and W. Krauth for helpful discussions. Support by the DFG through Re588/12 and the EU through TMR network "Patterns, Noise and Chaos", FMRX-CT96-0085 is gratefully acknowledged.
[1] R.M. Iverson, Rev. Geophys. 35, 245 (1997).

[2] M.H. Cooke, D.J. Stephens, and J. Bridgewater, Power Techol. 15, 1 (1976).

[3] A. Rosato, K.J. Stranburg, F. Prinz, and R.H. Swendsen, Phys. Rev. Lett. 58, 1038 (1987).

[4] M.J. Jaeger, R.S. Nagel, and R.P. Behringer, Rev. Mod. Phys. 68, 1259 (1996).

[5] O. Pouliquen, J. Delour, and S.B. Savage, Nature (London) 386, 816 (1997).

[6] F. Cantelaube and D. Bideau, Europhys. Lett. 30, 133 (1995).

[7] K.M. Hill and J.M. Kakalios, Phys. Rev. E 49, R3610 (1994).

[8] J. Duran, J. Rajchenbach, and E. Clément, Phys. Rev. Lett. 70, 2431 (1993).

[9] J.B. Knight, H.M. Jaeger, and S.R. Nagel, Phys. Rev. Lett. 70,
3728 (1993).

[10] S.B. Savage and C.K.K. Lun, J. Fluid Mech. 189, 311 (1988).

[11] A.D. Dinsmore, A.G. Yodh, and D.J. Pine, Phys. Rev. E 52, 4045 (1995).

[12] M.A. Scherer, V. Buchholtz, T. Pöschel, and I. Rehberg, Phys. Rev. E 54, R4560 (1996); M.A. Scherer et al., ibid. 61, 4069 (2000).

[13] S.L. Foerster, M.Y. Louge, H. Chang, and K. Allia, Phys. Fluids 6, 1108 (1994).

[14] L. D. Landau, and E. M. Lifshitz, Theory of Elasticity (Pergamon, Oxford, 1986).

[15] L. Kondic, Phys. Rev. E 60, 751 (1999); B. Painter, and R.P. Behringer, ibid. 62, 2380 (2000). 